\section{New developments}

\section{Benjamin G. Brackett}

Experimental Approaches to Mammalian Embryonic Development. Edited by Janet Rossant and Roger A. Pedersen. Cambridge University Press: 1987. Pp.558. £47.50, $\$ 70$.

"WHY study the mammalian embryo?" Janet Rossant and Roger A. Pedersen provide three answers in their preface to this book. First, as mammals ourselves, the study of other mammals can fulfil a need to learn more of our own development. Second, work with laboratory mammals has turned out to be directly beneficial in the development of human in vitro fertilization as a clinical procedure. Finally, mammals represent the last embryological frontier, one that is now accessible through new techniques.

The editors' intention was to review the large body of information now available on the cellular, biochemical and molecular aspects of the early mammalian embryo, and the exciting new approaches to developmental genetics made possible by recombinant DNA techniques. The resulting volume will be of value to anyone with an interest in the mammalian embryo: advanced undergraduates,

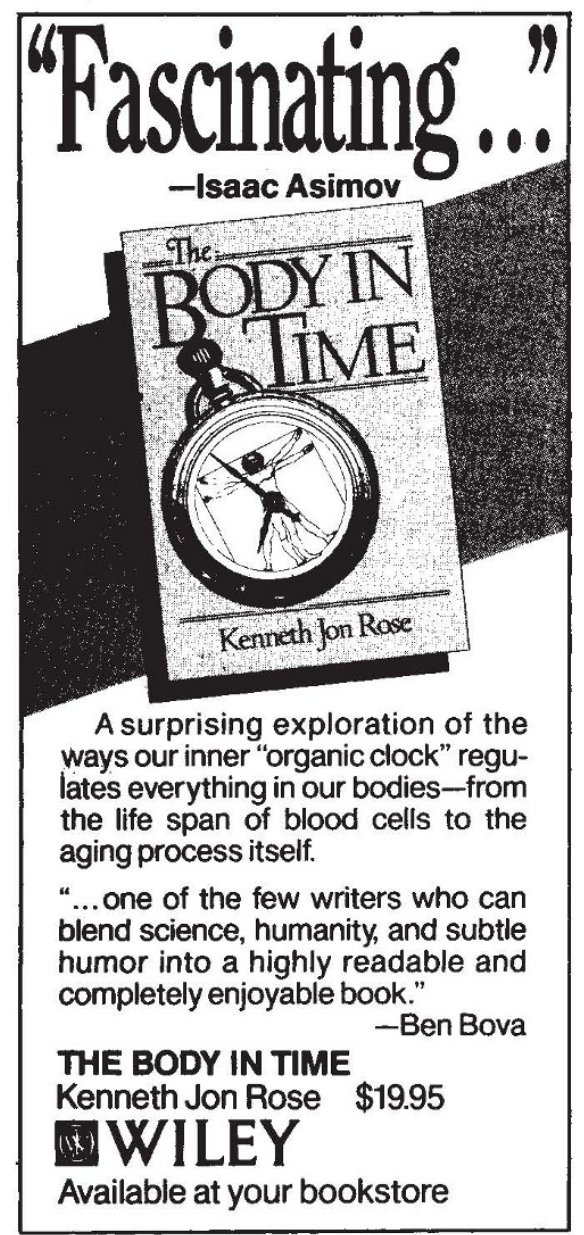

Reader Service No.2 graduate students, researchers with interests in developmental and molecular biology, and applied scientists with an eye on future applications. The reviews are well written and detailed, reflecting the expertise of each of the 21 authors, with literature cited up to early 1986 .

The book is divided into three general sections: cellular aspects, including studies of potency, allocation, differentiation, and fate in pre-implantation and early post-implantation embryos; molecular aspects, including gene expression during gametogenesis and early development, physiological aspects, cell surface and cytoskeletal differentiation, and $\mathrm{X}$-chromosome inactivation; and new approaches towards a genetic understanding of development, including nuclear transfer, analysis of genetic mutations, and introduction of new genetic material into the embryo via stem cell lines and production of transgenic mice.

One cannot peruse this volume without being struck by the overwhelming contribution of research on the laboratory mouse to our knowledge of early development. For me, however, those authors who depart from the mouse model

\section{By any other name}

\section{P.V.E. McClintock}

Dictionary of Effects and Phenomena in Physics. By Joachim Schubert. $\mathrm{VCH}$ : 1987. Pp.140. DM 54, £27.45.

Given the maturity of physics as a science and its continuing rate of expansion (almost two metres of new shelf-space per annum per library being needed for Physical Review alone), the relatively modest dimensions of Joachim Schubert's Dictionary of Effects and Phenomena in Physics come as something of a surprise. The main alphabetical listing runs to a mere 88 (smallish) pages, so that one is bound to question whether the subject has been covered: is it really all there?

The book is a new edition, in English, of an earlier German version. Its stated aim is to provide an overview for a broad readership of some 400 physical effects and phenomena, including historical notes about the discoveries and their discoverers together with references to the pertinent literature. The greater part of the volume consists of the dictionary itself, providing a succinct description of each effect or phenomenon and references to the original papers. There is also a series of 55 tables, dividing the phenomena into coherent groups, such as "low temperatures" or "magnetooptics" or "synergetic effects". A detailed chronology is provided, from " 1756 Leidenfrost phenomenon" up to "1984 JaccarinoPeter effect" and a select bibliography of represented an occasional and refreshing interlude. Thus the caution expressed by Virginia E. Papaioannou and Karl M. Ebert - "Although the broad outline of cell lineage relationships can be extrapolated using the mouse as a model, the details can be ascertained only by experimental studies on representative species" - seems particularly appropriate, not only in that context but to other aspects of the subject discussed in the book. Many of the approaches pioneered by work with the laboratory mouse point the way for studies in other mammals where important agricultural or medical applications are becoming increasingly obvious. The editors and authors are to be commended for their service in indicating such routes for future research. The objective of the editors was to summarize advances in mammalian embryology during the past decade, and they have succeeded. This book should indeed be a valuable addition to the library of "any serious student of the mammalian embryo".

Benjamin G. Brackett is a Professor in the Department of Physiology and Pharmacology, College of Veterinary Medicine, University of Georgia, Athens, Georgia 30602, USA.

\section{secondary sources (mostly books).}

Readers should be warned that one can sometimes fail to find entries relating to important phenomena. Usually, such entries are indeed included, but under unexpected headings and without there necessarily being cross-references from the more conventional terms. For example: "Quantum Hall effect" appears as "Von Klitzing effect", a perfectly reasonable appellation though not yet in common usage outside Germany; "Superfluidity" appears rather mysteriously under "Onnes effects", and it need imply no disrespect to the father of lowtemperature physics to point out that the phenomenon in question was in fact not identified as such until a decade after Onnes' death in 1926; the "AharonovBohm effect" is listed (not unreasonably but, in my experience, slightly unusually) as the "Bohm-Aharonov effect"; and so on. Thus, before abandoning the quest for any particular item, it is well worth considering alternatives and consulting the relevant table to see whether it has been included under a different name.

Although not quite the whole of physics is represented in the Dictionary, a very sizeable fraction of it certainly seems to be and the author is to be congratulated on encompassing so huge a mass of material in so small a space. The result is a handy reference work which should prove useful to many scientists and, in particular, to physicists straying outside their own particular specialisms.

P.V.E. McClintock is a Reader in the Department of Physics, University of Lancaster, Lancaster LAI $4 Y B, U K$. 